\title{
Acceleration of 2D-MR fingerprinting by reducing the number of echoes with increased in-plane resolution: a volunteer study
}

\author{
Yusuke Yokota ${ }^{1} \cdot$ Tomohisa Okada $^{2}$ - Yasutaka Fushimi ${ }^{1} \cdot$ Akira Yamamoto $^{1} \cdot$ Satoshi Nakajima ${ }^{1} \cdot$ Koji Fujimoto $^{2}$. \\ Sonoko Oshima ${ }^{1} \cdot$ Gregor Koerzdoerfer $^{3} \cdot$ Mathias Nittka $^{3} \cdot$ Josef Pfeuffer $^{3} \cdot$ Kaori Togashi $^{1}$
}

Received: 19 December 2019 / Revised: 22 March 2020 / Accepted: 24 March 2020 / Published online: 4 April 2020

(c) The Author(s) 2020

\begin{abstract}
Objective To compare the absolute values and repeatability of magnetic resonance fingerprinting (MRF) with 3000 and 1500 echoes/slice acquired in $41 \mathrm{~s}$ and $20 \mathrm{~s}$ (MRF3k and MRF1.5k, respectively).

Materials and methods MRF3k and MRF1.5k scans based on fast imaging with steady precession (FISP) were conducted using a $3 \mathrm{~T}$ scanner. Inter-scan agreement and intra-scan repeatability were investigated in 41 and 28 subjects, respectively. Region-of-interest (ROI) analysis was conducted on T1 values of MRF3k by two raters, and their agreement was evaluated using intraclass correlation coefficients (ICCs). Between MRF3k and MRF1.5k, differences in T1 and T2 values and inter-measurement correlation coefficients (CCs) were investigated. Intra-measurement repeatability was evaluated using coefficients of variation (CVs). A $p$ value $<0.05$ was considered statistically significant.

Results The ICCs of ROI measurements were 0.77-0.96. Differences were observed between the two MRF scans, but the CCs of the overall ROIs were 0.99 and 0.97 for the $\mathrm{T} 1$ and $\mathrm{T} 2$ values, respectively. The mean and median CVs of repeatability were equal to or less than $1.58 \%$ and $3.13 \%$ in each of the ROIs for T1 and T2, respectively; there were some significant differences between MRF3k and MRF1.5k, but they were small, measuring less than $1 \%$.

Discussion Both MRF3k and MRF1.5k had high repeatability, and a strong to very strong correlation was observed, with a trend toward slightly higher values in MRF1.5k.
\end{abstract}

Keywords Magnetic resonance imaging $\cdot$ Brain $\cdot$ Fingerprinting $\cdot$ Test-retest reliability

$\begin{array}{ll}\text { Abbreviations } \\ \text { CI } & \text { Confidence interval } \\ \text { CV } & \text { Coefficient of variation } \\ \text { FA } & \text { Flip angle } \\ \text { ICC } & \text { Intraclass correlation coefficient } \\ \text { MCP } & \text { Middle cerebellar peduncle }\end{array}$

Electronic supplementary material The online version of this article (https://doi.org/10.1007/s10334-020-00842-8) contains supplementary material, which is available to authorized users.

Tomohisa Okada

tomokada@kuhp.kyoto-u.ac.jp

1 Department of Diagnostic Imaging and Nuclear Medicine, Kyoto University, 54 Shogoin Kawaharacho, Sakyoku, Kyoto 606-8507, Japan

2 Human Brain Research Center, Graduate School of Medicine, Kyoto University, 54 Shogoin Kawaharacho, Sakyoku, Kyoto 606-8507, Japan

3 Magnetic Resonance, Siemens Healthcare GmbH, Henkestrasse 127 Postfach 32 60, 91050 Erlangen, Germany
MRF Magnetic resonance fingerprinting

ROI Region of interest

TE Echo time

TR Repetition time

\section{Introduction}

Magnetic resonance fingerprinting (MRF) is a recently introduced quantitative MRI framework that enables simultaneous measurement of multiple quantitative tissue parameters, such as T1 and T2 relaxation times. In MRF, acquisition parameters are varied in a pseudorandom manner so that different tissues have their own unique signal evolutions, which are compared with precalculated signal evolutions listed in a predefined dictionary to find the best match [1]. MRF has been used in brain disorders including brain tumors [2] and epilepsy [3,4] and is expected to enable better comparisons among different sites and scanners [5-7]. 
In MRF, a large number of measurements has been considered necessary to robustly generate parameter maps. One MRF scan of a single slice using the established method acquires 3000 echoes and takes 42 s [8], resulting in a 14-min scan for a whole-brain acquisition covering 20 slices; however, a faster and higher-resolution acquisition is favorable for clinical application. Current MRF measurements of $\mathrm{T} 1$ and T2 have been reported to be very stable [9, 10], and an MRF scan with a higher resolution in a shorter scan time is considered feasible.

Based on the results of a phantom study, we hypothesized that a 1500-echo MRF scan with some additional increase in spatial resolution would yield reliable measurements comparable to those of a 3000-echo MRF scan (i.e., twice the number of echoes). Therefore, the repeatability of the two MRF scans was compared, and agreement between them was investigated in a relatively large number of subjects.

\section{Materials and methods}

\section{Subjects}

This study was approved by the institutional review board. Forty-three healthy subjects (23 men, mean age 23.6 years, range 20-29 years) were enrolled in this study, and written informed consent was obtained before scanning. The exclusion criteria were poor image quality, which was typically due to excessive motion during scanning; apparent slice shifts; large susceptibility artifacts; and any abnormal findings in the brain. Two subjects were excluded due to large susceptibility artifacts caused by metal dental work affecting the values in the brain parenchyma, and MRF images of 41 subjects were used for further analysis.

\section{MR measurements}

A 3-T MR scanner (MAGNETOM Skyra, Siemens Healthcare, Erlangen, Germany) was used with a 32-channel head coil. Two-dimensional axial T2-weighted images parallel to the AC-PC plane were acquired with the following parameters: repetition time (TR), $5380 \mathrm{~ms}$, echo time (TE), $99 \mathrm{~ms}$; flip angle (FA), $150^{\circ}$, field of view, $230 \mathrm{~mm} \times 230 \mathrm{~mm}$; matrix size, $640 \times 640$; slice thickness, $5 \mathrm{~mm}$ with no interslice gaps. Based on the T2-weighted images, three slices showing the centrum semiovale, basal ganglia, and middle cerebellar peduncle (MCP) were selected, and MRF slices were placed at those slice positions. MRF scans were conducted using a prototype spiral fast imaging with steady precession (FISP) sequence [8] that has low sensitivity to magnetic field inhomogeneities. The trajectories were corrected using a one-time calibration and a generalized eddy-current model by Tan and Meyer [11]. Spiral trajectories were rotated by $82.5^{\circ}$ between successive repetitions [12].

The slices were measured in a sequential, non-interleaved manner. Each slice acquisition consisted of an adiabatic, non-selective inversion pulse and a train of FISP echoes. Acquisition started at $21 \mathrm{~ms}$ after the inversion pulse with a TE of $2 \mathrm{~ms}$ and a base TR of $12 \mathrm{~ms}$. TR and FA were continuously changed, ranging from 12.1 to $15.0 \mathrm{~ms}$ and from $0^{\circ}$ to $74^{\circ}$, respectively (Fig. 1). MRF with 3000 echoes (MRF3k) [8, 10] and MRF with 1500 echoes (MRF1.5k) were conducted. The latter scan parameter was set based on a study using an NIST/ISMRM system phantom (https:// www.nist.gov/programs-projects/quantitative-mri). Details are presented in the supplementary material.

The scan time of each slice was $41 \mathrm{~s}$ and $20 \mathrm{~s}$ for MRF3k and MRF1.5k, respectively. For MRF3k, the field of view was $300 \mathrm{~mm} \times 300 \mathrm{~mm}$, and the in-plane resolution was $1.17 \mathrm{~mm} \times 1.17 \mathrm{~mm}$, for MRF1.5k, the values were $256 \mathrm{~mm} \times 256 \mathrm{~mm}$ and $1.0 \mathrm{~mm} \times 1.0 \mathrm{~mm}$, respectively.
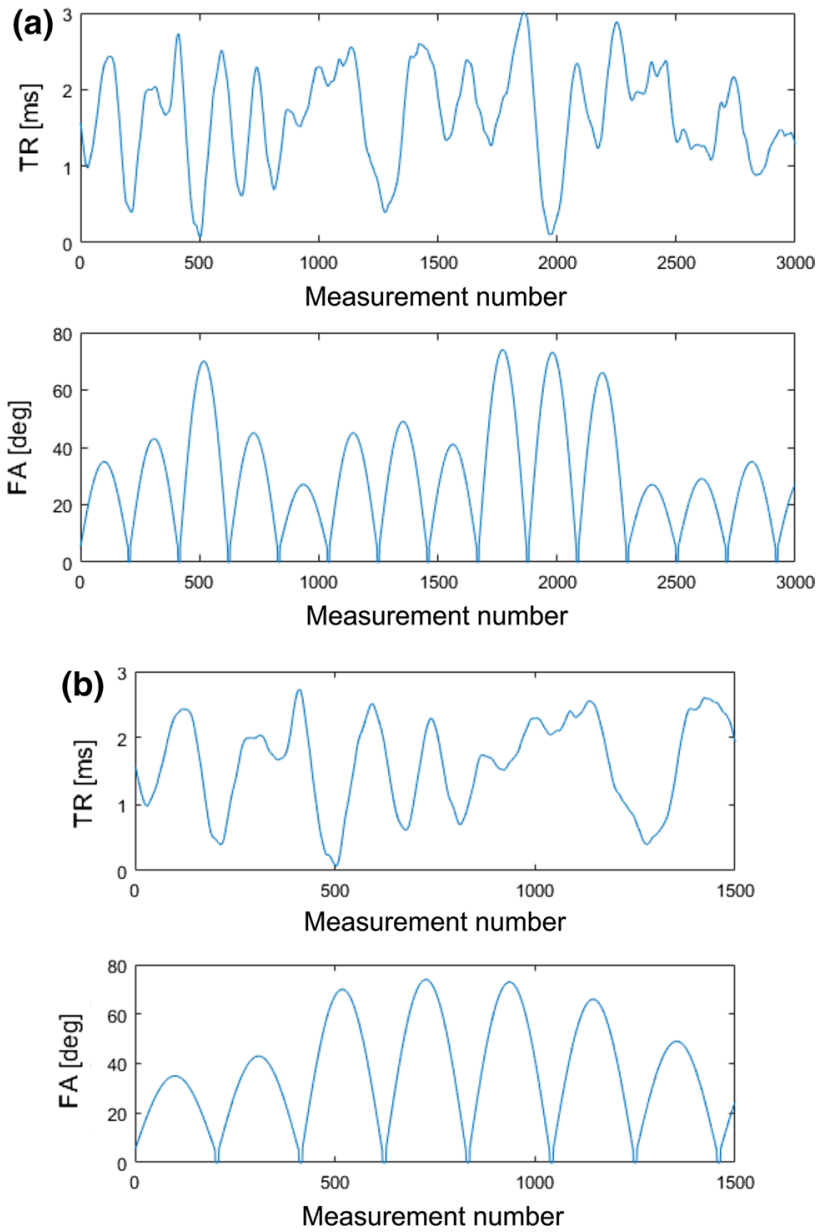

Fig. 1 TRs and FAs for a MRF3k and b MRF1.5k. Changes in TR are the same, but different FA patterns were used for the two measurements 
The slice thickness was $5 \mathrm{~mm}$ for both scans. Before the MRF scans, an RF-field map [13] of the whole volume was acquired in $20 \mathrm{~s}$ and used during the reconstruction of both MRF scans. Each MRF scan was repeated twice in 28 out of the 41 subjects. Representative images are presented in Fig. 2.

The MRF dictionary was calculated for a range of discrete $\mathrm{T} 1$ and $\mathrm{T} 2$ values using the Bloch equation. The step sizes (ranges: min-max) were $10 \mathrm{~ms}(10-100 \mathrm{~ms})$, $20 \mathrm{~ms}(100-1000 \mathrm{~ms}), 40 \mathrm{~ms}(1000-2000 \mathrm{~ms})$ and $100 \mathrm{~ms}$ $(2000-4500 \mathrm{~ms})$ for $\mathrm{T} 1$ and $2 \mathrm{~ms}(2-100 \mathrm{~ms}), 5 \mathrm{~ms}$ (100-150 ms), $10 \mathrm{~ms}(150-300 \mathrm{~ms}), 50 \mathrm{~ms}(300-800 \mathrm{~ms})$, $100 \mathrm{~ms}(800-1600 \mathrm{~ms})$ and $200 \mathrm{~ms}(1600-3000 \mathrm{~ms})$ for T2, which were the same for both MRF3k and MRF1.5k.

\section{Image pre-processsing and ROI analyses}

After brain extraction, MRF1.5k T1 and T2 maps were registered to the MRF3k T1 map of the same slice for each subject in 2D mode (in-plane translation and rotation) and resampled to $1.17 \mathrm{~mm} \times 1.17 \mathrm{~mm}$ in-plane resolution using FMRIB's Linear Image Registration Tool (https://fsl.fmrib .ox.ac.uk/fsl/fslwiki).

The following structures were selected for region-ofinterest (ROI) analysis: centrum semiovale, caudate head, putamen, globus pallidus, thalamus, corpus callosum, middle cerebellar peduncle (MCP), and ventral part of the pons (Fig. 3). ROIs on the standard MRF3k T1 map images were drawn independently by two raters (rater 1: Y.Y. with 11 years and rater 2: S.N. with 15 years of experience as neuroradiologists) using ImageJ software (https://imagej.nih. gov/ij/). For some of the structures with apparent boundaries, i.e., the basal ganglia, caudate head, and thalamus, ROIs were drawn by tracing their inner boundaries. For the rest of the structures with no apparent boundaries, circular ROIs were placed. The mean $\mathrm{T} 1$ and $\mathrm{T} 2$ values of the ROIs were measured.

\section{Statistical analyses}

The Shapiro-Wilk test was used to check the normality of the data distribution.

Inter-rater agreement in ROI placement was evaluated using the intraclass correlation coefficient (ICC) of the mean $\mathrm{T} 1$ values of two raters' ROIs positioned on the MRF3k T1 maps. The ICC value is interpreted as a reliability scale in the following manner: less than 0.5 , poor; $0.5-0.75$, moderate; 0.75-0.9, good; greater than 0.90, excellent [14].

The inter-measurement differences in the mean $\mathrm{T} 1$ and $\mathrm{T} 2$ values of each of the ROIs were examined between MRF3k
Fig. 2 Representative MRF images of a subject

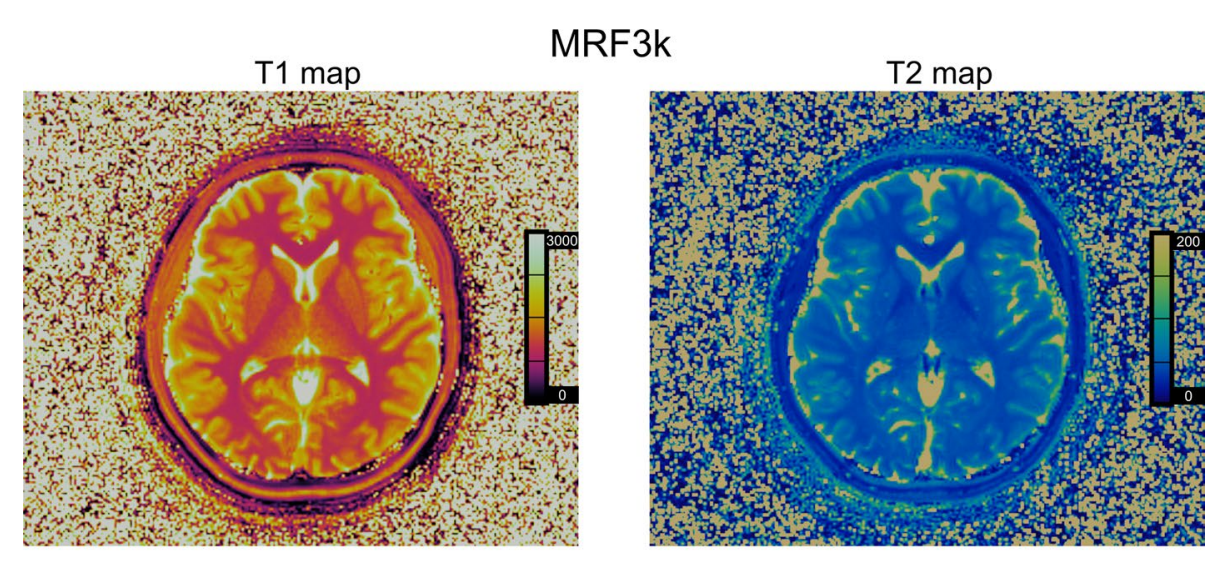

T1 map

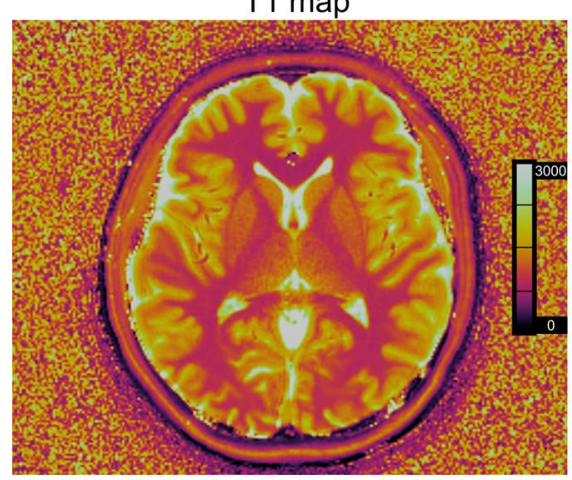

MRF1.5k

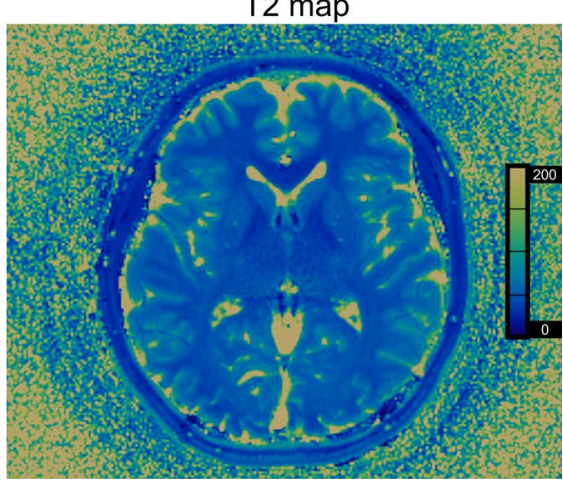




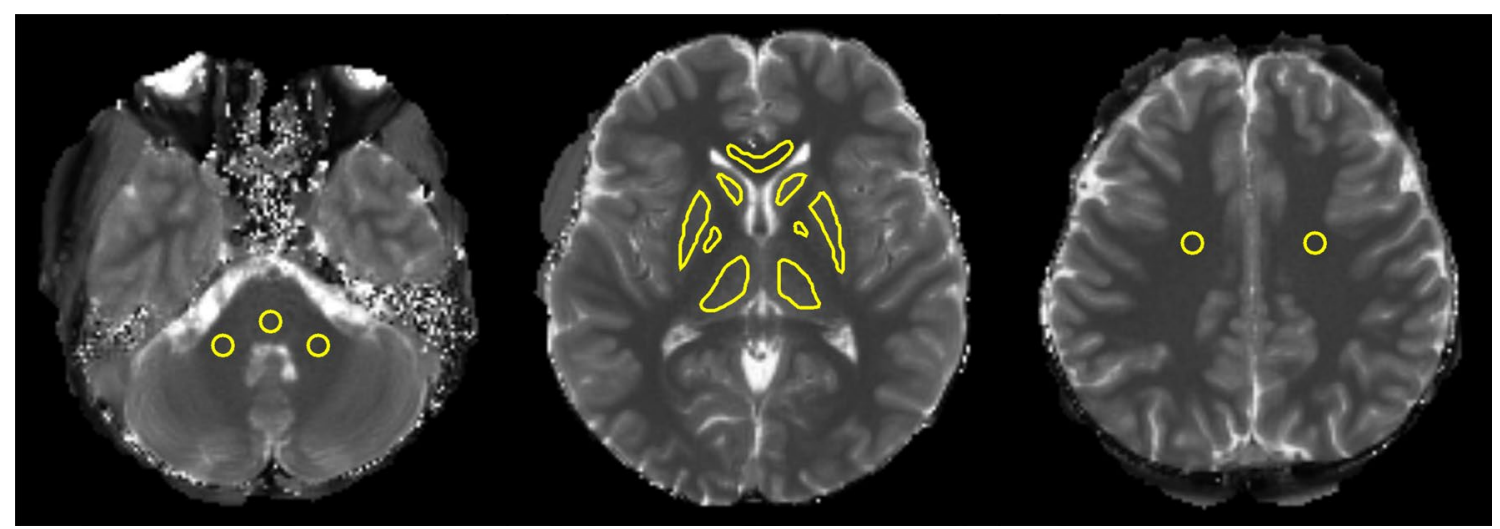

Fig. 3 Multiple ROIs placed for analysis. ROIs were located in a the MCP, pons, b caudate head, putamen, globus pallidus, thalamus, corpus callosum, and $\mathbf{c}$ bilateral centrum semiovale

and MRF1.5k using paired $t$ tests or Wilcoxon tests. Intermeasurement correlation analysis was also conducted using Pearson's correlation coefficient or Spearman's rank correlation coefficient. In the cases with repeated measurements, the mean of two measurement values was used. Biases of T1 and T2 values between MRF3k and MRF1.5k were assessed using Bland-Altman plots.

Intra-measurement repeatability was evaluated using coefficients of variation (CVs) of mean $\mathrm{T} 1$ and $\mathrm{T} 2$ values of repeated measurements for both MRF3k and MRF1.5k using the abovementioned ROIs. These CVs were compared between MRF3k and MRF1.5k using paired $t$ test or Wilcoxon test.

Statistical significance was defined as $p<0.05$. All statistics were calculated using MedCalc Statistical Software version 18 (MedCalc Software bvba, Ostend, Belgium).
Table 1 Intra-class correlation coefficient (ICC) of T1 values derived from 2 raters' ROIs

and 1024-1381 ms and 1038-1397 ms, respectively, in the gray matter (caudate, basal ganglia and thalamus). The T2 values in MRF3k and MRF1.5k were 32.7-38.3 ms and 34.0-41.0 ms, respectively, at the cerebral white matter ROIs and 29.1-51.7 $\mathrm{ms}$ and 31.1-53.9 ms, respectively, in the deep gray matter ROIs. Some ROI values showed significant inter-measurement differences (Table 2).

Between MRF3k and MRF1.5k, the correlation coefficients of the measured T1 and T2 values of each ROI were $0.79-0.98$ and $0.73-0.93$, respectively, showing a strong to very strong correlation [15], and those of the overall ROI values were 0.99 and 0.97 for the T1 and T2 values, respectively (Table 3 ). Bland-Altman plots of T1 and $\mathrm{T} 2$ values showed relatively small differences in the 
Table $2 \mathrm{~T} 1$ and T2 values in regions-of-interest (ROIs) of rater 1

\begin{tabular}{|c|c|c|c|c|c|}
\hline \multirow[t]{2}{*}{ ROIs } & \multicolumn{2}{|l|}{ MRF3k } & \multicolumn{2}{|l|}{ MRF1.5k } & \multirow[t]{2}{*}{$p$ value } \\
\hline & $\mathrm{T} 1$ value & $95 \% \mathrm{CI}$ & T1 value & $95 \% \mathrm{CI}$ & \\
\hline Left MCP & 1022 & $1012-1032$ & 1032 & $1020-1044$ & $0.003 *$ \\
\hline Right MCP & 1044 & $1034-1054$ & 1045 & $1034-1055$ & 0.875 \\
\hline Pons & $1100^{\dagger}$ & $1084-1111^{\dagger}$ & $1123^{\dagger}$ & $1113-1138^{\dagger}$ & $<0.001^{\dagger *}$ \\
\hline Left caudate & 1381 & $1369-1394$ & 1397 & $1385-1409$ & $<0.001 *$ \\
\hline Right caudate & 1349 & $1337-1362$ & 1373 & $1359-1386$ & $<0.001^{*}$ \\
\hline Left putamen & 1244 & $1233-1256$ & 1263 & $1252-1274$ & $<0.001 *$ \\
\hline Right putamen & 1226 & $1214-1237$ & 1256 & $1245-1267$ & $<0.001^{*}$ \\
\hline Left pallidus & $1053^{\dagger}$ & $1032-1067^{\dagger}$ & $1038^{\dagger}$ & $1015-1054^{\dagger}$ & $0.002^{\dagger *}$ \\
\hline Right pallidus & 1024 & $1013-1036$ & 1039 & $1028-1051$ & $<0.001 *$ \\
\hline Left thalamus & 1167 & $1148-1186$ & 1168 & $1148-1188$ & 0.595 \\
\hline Right thalamus & 1150 & $1133-1168$ & 1171 & $1152-1190$ & $<0.001^{*}$ \\
\hline Corpus callosum & 870 & $861-879$ & 862 & $852-872$ & $0.006^{*}$ \\
\hline Left semiovale & $919^{\dagger}$ & $907-925^{\dagger}$ & $910^{\dagger}$ & $897-923^{\dagger}$ & $0.001^{\dagger *}$ \\
\hline$\underline{\text { Right semiovale }}$ & $903^{\dagger}$ & 889-918 & $904^{\dagger}$ & 889-918 & $0.059^{\dagger}$ \\
\hline \multirow[t]{2}{*}{ ROIs } & \multicolumn{2}{|l|}{ MRF3k } & \multicolumn{2}{|l|}{ MRF1.5k } & \multirow[t]{2}{*}{$p$ value } \\
\hline & $\mathrm{T} 2$ value & $95 \% \mathrm{CI}$ & $\mathrm{T} 2$ value & $95 \% \mathrm{CI}$ & \\
\hline Left MCP & $39.9^{\dagger}$ & $38.4-41.9^{\dagger}$ & $43.7^{\dagger}$ & $42.2-45.4^{\dagger}$ & $<0.001^{\dagger * *}$ \\
\hline Right MCP & $40.2^{\dagger}$ & $39.4-41.1^{\dagger}$ & 43.3 & $42.5-44.2^{\dagger}$ & $<0.001^{\dagger *}$ \\
\hline Pons & $38.1^{\dagger}$ & $36.2-40.0^{\dagger}$ & $41.4^{\dagger}$ & $40.0-43.3^{\dagger}$ & $<0.001^{\dagger *}$ \\
\hline Left caudate & 50.9 & $50.1-51.7$ & 52.8 & $52.0-53.5$ & $<0.001 *$ \\
\hline Right caudate & 51.7 & $50.9-52.4$ & 53.9 & $53.1-54.6$ & $<0.001^{*}$ \\
\hline Left putamen & $45.1^{\dagger}$ & $44.2-45.7^{\dagger}$ & $47.9^{\dagger}$ & $47.3-48.5^{\dagger}$ & $<0.001^{\dagger *}$ \\
\hline Right putamen & 45.4 & $44.6-46.3$ & 48.8 & $48.0-49.7$ & $<0.001 *$ \\
\hline Left pallidus & $29.6^{\dagger}$ & $29.1-30.2^{\dagger}$ & $31.1^{\dagger}$ & $30.4-31.5^{\dagger}$ & $<0.001^{\dagger *}$ \\
\hline Right pallidus & 29.1 & $28.4-29.7$ & 31.4 & $30.8-32.1$ & $<0.001^{*}$ \\
\hline Left thalamus & 41.8 & $41.1-42.4$ & 44.3 & $43.5-45.0$ & $<0.001 *$ \\
\hline Right thalamus & $41.4^{\dagger}$ & $40.5-42.0^{\dagger}$ & $44.4^{\dagger}$ & $43.8-45.4^{\dagger}$ & $<0.001^{\dagger *}$ \\
\hline Corpus callosum & 32.7 & $32.0-33.5$ & 34.0 & $33.3-34.7$ & $<0.001 *$ \\
\hline Left semiovale & $38.1^{\dagger}$ & $37.2-39.1^{\dagger}$ & $41.0^{\dagger}$ & $40.0-41.8^{\dagger}$ & $<0.001^{\dagger *}$ \\
\hline Right semiovale & $38.3^{\dagger}$ & $37.2-39.2^{\dagger}$ & $40.1^{\dagger}$ & $39.7-40.9^{\dagger}$ & $<0.001^{\dagger *}$ \\
\hline
\end{tabular}

$C I$ confidence interval, $M C P$ middle cerebellar peduncle

*Statistical significance

${ }^{\dagger}$ Non-normal distribution, and median values are presented, and non-parametric Wilcoxon test was used for comparison

mean percent differences $(95 \% \mathrm{CI})$ of 0.9 (- 3.0 to 4.8 ) and 6.6 ( -0.8 to 14.0 ) for T1 and T2 values, respectively, between MRF3k and MRF1.5k (Fig. 4).

\section{Intra-measurement repeatability}

The mean or median CVs of repeatedly measured $\mathrm{T} 1$ values of each ROI in 28 subjects were $0.45-1.31 \%$ in MRF3k and $0.40-1.58 \%$ in MRF1.5k. The CV of T1 values was significantly higher in MRF1.5k than MRF3k in the right MCP, but the difference was only $0.65 \%$. The mean or median CVs of $\mathrm{T} 2$ values were $0.55-2.50 \%$ and $0.90-3.13 \%$ for MRF3k and MRF1.5k, respectively. The
CVs of T2 values were significantly higher in the left putamen, left thalamus and right thalamus in MRF1.5k than in MRF3k, but the differences were only $0.69,0.98$ and $0.85 \%$, respectively (Table 4). Differences in CVs measured as an index of repeatability were found to be less than $1 \%$ in each of the ROIs.

\section{Discussion}

In this study, MRF measurements with different numbers of echoes, and consequently scan lengths, were compared to examine inter-measurement differences and correlations of 
Table 3 Inter-measurement correlation coefficient $(r)$ and 95\% confidence interval (CI) between MRF3k and MRF1.5k

\begin{tabular}{|c|c|c|c|c|}
\hline \multirow[t]{2}{*}{ ROIs } & \multicolumn{2}{|c|}{$\mathrm{T} 1$ value } & \multicolumn{2}{|c|}{$\mathrm{T} 2$ value } \\
\hline & $r$ & $95 \% \mathrm{CI}$ & $r$ & $95 \% \mathrm{CI}$ \\
\hline Left MCP & 0.88 & $0.78-0.93$ & 0.83 & $0.71-0.91$ \\
\hline Right MCP & 0.84 & $0.71-0.91$ & $0.85^{\dagger}$ & $0.73-0.91$ \\
\hline Pons & 0.81 & $0.66-0.89$ & 0.92 & $0.85-0.96$ \\
\hline Left caudate & 0.83 & $0.69-0.90$ & 0.88 & $0.78-0.93$ \\
\hline Right caudate & 0.79 & $0.64-0.88$ & 0.73 & $0.54-0.84$ \\
\hline Left putamen & 0.88 & $0.78-0.93$ & 0.90 & $0.82-0.95$ \\
\hline Right putamen & 0.92 & $0.86-0.96$ & 0.93 & $0.87-0.96$ \\
\hline Left pallidus & 0.80 & $0.65-0.89$ & $0.88^{\dagger}$ & $0.79-0.94$ \\
\hline Right pallidus & 0.88 & $0.79-0.94$ & 0.90 & $0.82-0.95$ \\
\hline Left thalamus & 0.98 & $0.97-0.99$ & 0.88 & $0.78-0.93$ \\
\hline Right thalamus & 0.97 & $0.95-0.99$ & 0.89 & $0.80-0.94$ \\
\hline Corpus callosum & 0.86 & $0.74-0.92$ & 0.83 & $0.71-0.91$ \\
\hline Left semiovale & 0.80 & $0.66-0.89$ & 0.77 & $0.60-0.87$ \\
\hline Right semiovale & 0.87 & $0.76-0.93$ & 0.79 & $0.63-0.88$ \\
\hline Overall ROIs & $0.99^{\dagger}$ & $0.99-0.99$ & $0.97^{\dagger}$ & $0.97-0.98$ \\
\hline
\end{tabular}

$M C P$ middle cerebellar peduncle, $R O I s$ regions-of-interest

${ }^{\dagger}$ Non-normal distribution, and median values are presented, and nonparametric Spearman's rank correlation coefficient was used for comparison

absolute T1 and T2 values using a relatively large number of subjects. The mean T1 and T2 values in this study were similar to those in a multi-center MRF study [10]. The values were strongly to very strongly correlated between MRF3k and MRF1.5k. However, the estimated T1 and T2 values were higher by $0.9 \%$ and $6.6 \%$, respectively, in MRF1.5k than in MRF3k. Considering these biases, either one type of MRF should be consistently used to take advantage of the high repeatability of MRF scans presented in this study.

$\mathrm{CVs}$, an index of intra-measurement repeatability, were less than $1.58 \%$ for $\mathrm{T} 1$ and $3.13 \%$ for $\mathrm{T} 2$. The CVs were significantly higher at the right MCP for T1 and at the left putamen and bilateral thalamus for $\mathrm{T} 2$ in MRF1.5k than in MRF3k, but the differences in CVs were less than $1 \%$ (see Table 4). These results confirmed that cutting the scan time in half has little effect on the repeatability. However, it should be noted that the acquisition parameters of MRF1.5k were not exactly the same as the first half of MRF3k. The same TR values were used, but the FAs were higher in MRF1.5k than in MRF3k (see Fig. 1). In addition, MRF1.5k had a slightly higher in-plane resolution that is considered advantageous, especially for small structures such as the substantia nigra, red nucleus, subthalamic nucleus, and habenula. However, this potential advantage was not exploited in the comparison with MRF3k because MRF1.5k images were down-sampled to match the resolution of
MRF3k. The original pixel size of the MRF1.5k images was approximately $27 \%$ smaller $\left(1-1.0^{2} / 1.17^{2}\right)$, resulting in a lower signal-to-noise ratio (SNR), and ROIs were placed on the MRF3k T1 maps. The coregistration of MRF1.5k images to MRF3k images was a linear transform with inplane translation and rotation. Resampling was conducted using bilinear interpolation, which will introduce a certain averaging effect, i.e., possible increase in SNR, but the average values of the ROI are the same except for the pixels at the edge of ROIs. Therefore, the effect of resampling on $\mathrm{SNR}$ is considered small. These procedures possibly caused higher CVs in MRF1.5k than in MRF3k.

Recently, a multi-center study that assessed the repeatability and reproducibility of 2D MRF using FISP, which is the same as MRF3k but used a 20-channel head coil, was reported [10]. Intra-scanner repeatability half-widths of the confidence intervals for relative deviations, which are calculated as 1.96 times the CVs, were in the range of $2.0-3.1 \%$ for $\mathrm{T} 1$ and $3.1-7.9 \%$ for $\mathrm{T} 2$ in solid tissue compartments. The CVs of MRF3k were $0.45-1.31 \%$ and $0.55-2.50 \%$ for $\mathrm{T} 1$ and $\mathrm{T} 2$ values, respectively, and our results concerning intra-scanner repeatability half-widths were $0.88-2.56 \%$ and $1.08-3.61 \%$, showing similar high repeatability. The $\mathrm{CV}$ s of $\mathrm{T} 2$ were higher than those of $\mathrm{T} 1$, apparently due to the relatively narrow ranges of $\mathrm{TR}$ and $\mathrm{TE}$, which reduce T2-related signal changes. Such limitations of this type of 2D-MRF measurement caused T2 to have lower repeatability than $\mathrm{T} 1$.

In a prior study, T2 measurements had greater variation than T1 measurements [9]. This difference was attributed to $\mathrm{B} 1$ variation that affects the measured T2 values more than T1 values in a previous MRF study [16]. Diffusion weighting caused by spoiler gradients may also lead to inaccurate T2 measurements in MRF [17]. However, both B1 and spoiler gradients were the same for MRF3k and MRF1.5k. These factors also explain the larger CVs in $\mathrm{T} 2$ than $\mathrm{T} 1$ but not for bias. Although it cannot be accounted for, no apparent trend was observed dependent on the averaged $\mathrm{T} 2$ values, and the bias was stable. These results support the consistent use of either MRF3k or MRF1.5k.

The proposed MRF1.5k scan reduced the scan time by half while retaining high repeatability comparable to that of the long scan, i.e., MRF3k [10]. This large reduction in scan time is highly advantageous in clinical practice. Quantitative imaging biomarkers are increasingly employed to investigate pathogenic processes and monitor therapeutic response [18], and MRF is expected to play an important role. Further reduction in scan time by a simultaneous multi-slice [19] and 3D [20] MRF as well as advanced reconstruction methods [21-23] will facilitate the use of MRF.

Our study has some limitations. First, no relaxometry method other than MRF was used to compare the absolute 

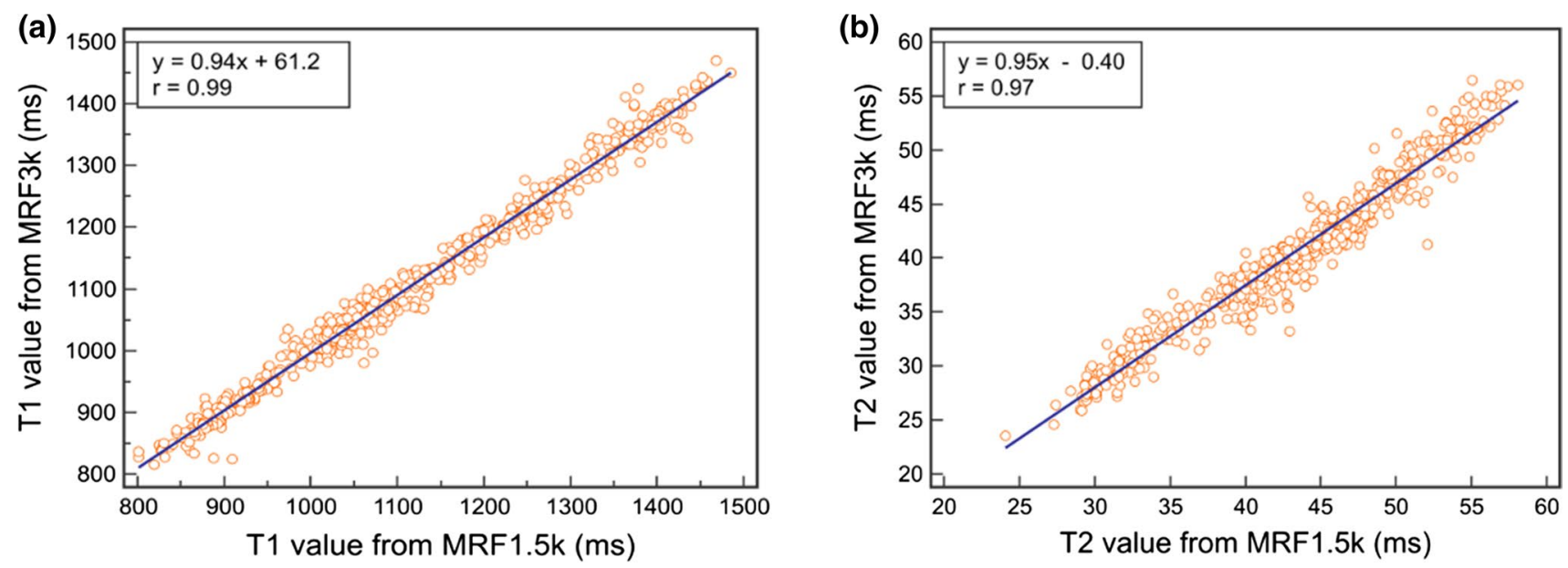

(c)

(d)
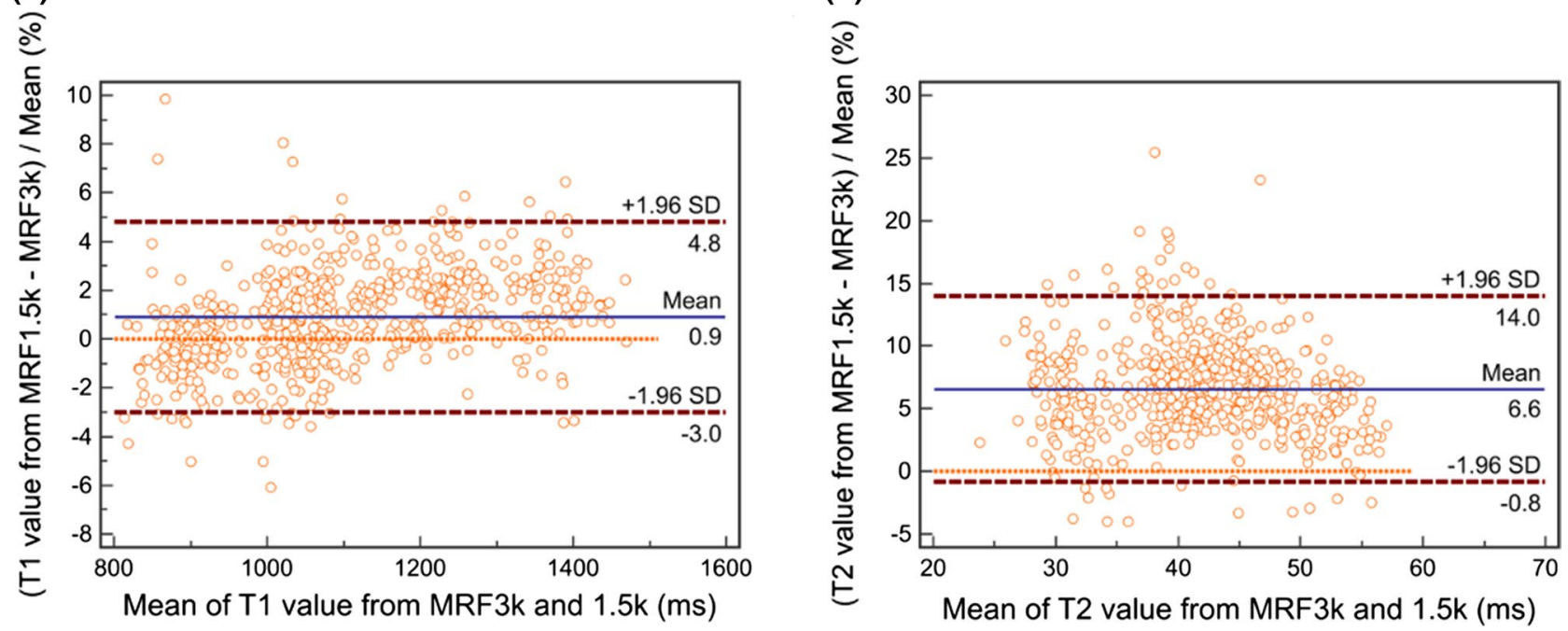

Fig. 4 Correlation plots (top, $\mathbf{a}$ and $\mathbf{b}$ ) and Bland-Altman plots (bottom, $\mathbf{c}$ and $\mathbf{d}$ ) of overall ROI values between MRF3k and MRF1.5k for (right) $\mathrm{T} 1$ and (left) $\mathrm{T} 2$ values

values. Second, the alignment of each image was performed in 2D mode so that slight subject motion could be corrected and the same ROIs could be applied for objective analysis; if a subject's brain was shifted in up/down or rotated in the pitch or roll direction during imaging, such displacement could not be corrected, but no apparent misregistration was noticed by the raters. Third, we compared 4 scan conditions-echo numbers of 3000 vs. 1500 and in-plane resolution of $1.17 \mathrm{~mm} \times 1.17 \mathrm{~mm}$ vs. $1 \mathrm{~mm} \times 1 \mathrm{~mm}$-in the phantom (see supplementary material) but not in the human subjects. This was because the
4 scan conditions were comparable in the phantom, and we intended to focus on MRF3k $(1.17 \mathrm{~mm} \times 1.17 \mathrm{~mm})$ and MRF1.5k $(1 \mathrm{~mm} \times 1 \mathrm{~mm})$ to reduce the total scan time and compare them in a relatively large number of human subjects. Fourth, ROIs may introduce rater biases, but ICCs between raters were good to excellent (0.77-0.98), and such biases are considered limited.

In conclusion, MRF3k and MRF1.5k had high repeatability and were strongly correlated this healthy-volunteer study; however, some measurement bias suggests that consistent use of one condition or the other would be optimal. 
Table 4 Mean and median coefficient of variation (CVs) for intra-measurement repeatability of MRF3k and MRF1.5k and the 95\% confidence intervals (CI) as well as statistical difference ( $p$ values) for T1 (a) and T2 (b) values

\begin{tabular}{|c|c|c|c|c|c|}
\hline \multirow[t]{2}{*}{ ROIs } & \multicolumn{2}{|l|}{ MRF3k } & \multicolumn{2}{|l|}{ MRF1.5k } & \multirow[t]{2}{*}{$p$ value } \\
\hline & CVs of $\mathrm{T} 1$ & $95 \% \mathrm{CI}$ & CVs of T1 & $95 \% \mathrm{CI}$ & \\
\hline \multicolumn{6}{|l|}{ (a) } \\
\hline Left MCP & 0.84 & $0.58-1.10$ & 1.04 & $0.74-1.33$ & 0.29 \\
\hline Right MCP & 0.8 & $0.60-0.99$ & 1.45 & $1.04-1.86$ & $0.004 *$ \\
\hline Pons & 1.31 & $0.89-1.72$ & 0.83 & $0.59-1.07$ & 0.06 \\
\hline Left caudate & 0.95 & $0.63-1.27$ & 0.91 & $0.58-1.23$ & 0.85 \\
\hline Right caudate & 0.95 & $0.65-1.24$ & 0.94 & $0.70-1.18$ & 0.97 \\
\hline Left putamen & 0.59 & $0.42-0.77$ & 0.76 & $0.54-0.98$ & 0.26 \\
\hline Right putamen & 0.51 & $0.37-0.64$ & 0.66 & $0.47-0.86$ & 0.17 \\
\hline Left pallidus & 1.11 & $0.76-1.46$ & 1.58 & $1.21-1.94$ & 0.09 \\
\hline Right pallidus & 0.94 & $0.60-1.27$ & 1.15 & $0.85-1.46$ & 0.34 \\
\hline Left thalamus & 0.66 & $0.44-0.88$ & 0.63 & $0.45-0.82$ & 0.83 \\
\hline Right thalamus & $0.45^{\dagger}$ & $0.30-0.70^{\dagger}$ & $0.40^{\dagger}$ & $0.30-0.80^{\dagger}$ & $0.48^{\dagger}$ \\
\hline Corpus callosum & 1.1 & $0.72-1.48$ & 1.13 & $0.76-1.50$ & 0.91 \\
\hline Left semiovale & $0.50^{\dagger}$ & $0.34-0.70^{\dagger}$ & $0.75^{\dagger}$ & $0.54-1.09^{\dagger}$ & $0.07^{\dagger}$ \\
\hline Right semiovale & 0.66 & $0.44-0.88$ & 0.86 & $0.56-1.16$ & 0.07 \\
\hline \multirow[t]{2}{*}{ ROIs } & \multicolumn{2}{|l|}{ MRF3k } & \multicolumn{2}{|l|}{ MRF1.5k } & $p$ value \\
\hline & $\mathrm{CVs}$ of $\mathrm{T} 2$ & $95 \% \mathrm{CI}$ & CVs of $\mathrm{T} 2$ & $95 \% \mathrm{CI}$ & \\
\hline \multicolumn{6}{|l|}{ (b) } \\
\hline Left MCP & 1.63 & $1.06-2.20$ & 2.09 & $1.63-2.55$ & 0.27 \\
\hline Right MCP & 1.48 & $1.06-1.89$ & 1.47 & $0.98-1.96$ & 0.98 \\
\hline Pons & 2.50 & $1.85-3.15$ & 3.13 & $2.31-3.95$ & 0.25 \\
\hline Left caudate & 1.14 & $0.65-1.62$ & 1.74 & $1.16-2.32$ & 0.08 \\
\hline Right caudate & 1.71 & $1.13-2.28$ & 2.26 & $1.48-3.05$ & 0.21 \\
\hline Left putamen & 0.79 & $0.39-1.19$ & 1.48 & $1.00-1.95$ & $0.02 *$ \\
\hline Right putamen & 1.06 & $0.68-1.44$ & 1.08 & $0.70-1.45$ & 0.95 \\
\hline Left pallidus & 1.84 & $1.07-2.62$ & 2.18 & $1.52-2.83$ & 0.44 \\
\hline Right pallidus & 1.36 & $0.95-1.78$ & 1.78 & $1.24-2.31$ & 0.29 \\
\hline Left thalamus & 0.99 & $0.65-1.33$ & 1.97 & $1.38-2.56$ & $0.01 *$ \\
\hline Right thalamus & $0.55^{\dagger}$ & $0.30-1.26^{\dagger}$ & $1.40^{\dagger}$ & $0.94-2.10^{\dagger}$ & $0.001^{\dagger *}$ \\
\hline Corpus callosum & 1.64 & $1.07-2.20$ & 2.10 & $1.49-2.72$ & 0.22 \\
\hline Left semiovale & $0.65^{\dagger}$ & $0.40-1.10^{\dagger}$ & $1.00^{\dagger}$ & $0.74-1.60^{\dagger}$ & $0.33^{\dagger}$ \\
\hline Right semiovale & $0.70^{\dagger}$ & $0.44-0.86^{\dagger}$ & $0.90^{\dagger}$ & $0.74-1.40^{\dagger}$ & $0.24^{\dagger}$ \\
\hline
\end{tabular}

$M C P$ middle cerebellar peduncle, $R O I s$ regions-of-interest

*Asterisks indicate statistical significance

†Obelisks indicate non-normal distribution, and median values are presented, and non-parametric Wilcoxon test was used for comparison

Author contribution statement YY: acquisition of data, analysis and interpretation of data, drafting of manuscript. TO: conception and design of study, analysis and interpretation of data, drafting of manuscript. YF: analysis and interpretation of data, critical revision. AY: analysis and interpretation of data, critical revision. SN: analysis and interpretation of data, critical revision. KF: critical revision. SO: analysis and interpretation of data, critical revision. GK: creation of MRF software, critical revision. MN: creation of MRF software, critical revision. JP: creation of MRF software, critical revision. KT: conception and design of study, critical revision.
Funding This study was partly funded by the Japan Society for the Promotion of Science Challenging Research (Exploratory) Grant to Author T.O. (Grant Number 18K18453).

\section{Compliance with ethical standards}

Conflict of interest Authors G.K., M.N., and J.P. are employees of Siemens Healthcare GmbH. Author T.O. has received a research grant for 7 T MR from Siemens Healthcare K.K. The other authors declare that they have no conflict of interest. 
Ethical approval All procedures performed in studies involving human participants were in accordance with the ethical standards of the institutional and/or national research committee (Kyoto University Graduate School and Faculty of Medicine, Kyoto University Hospital Ethics Committee: R1487) and with the 1964 Declaration of Helsinki and its later amendments or comparable ethical standards.

Informed consent Informed consent was obtained from all individual participants included in the study.

Open Access This article is licensed under a Creative Commons Attribution 4.0 International License, which permits use, sharing, adaptation, distribution and reproduction in any medium or format, as long as you give appropriate credit to the original author(s) and the source, provide a link to the Creative Commons licence, and indicate if changes were made. The images or other third party material in this article are included in the article's Creative Commons licence, unless indicated otherwise in a credit line to the material. If material is not included in the article's Creative Commons licence and your intended use is not permitted by statutory regulation or exceeds the permitted use, you will need to obtain permission directly from the copyright holder. To view a copy of this licence, visit http://creativecommons.org/licenses/by/4.0/.

\section{References}

1. Ma D, Gulani V, Seiberlich N, Liu K, Sunshine JL, Duerk JL, Griswold MA (2013) Magnetic resonance fingerprinting. Nature 495:187-192

2. Badve C, Yu A, Dastmalchian S, Rogers M, Ma D, Jiang Y, Margevicius S, Pahwa S, Lu Z, Hluchter M, Sunshine J, Griswold M, Sloan A, Gulani V (2016) MR Fingerprinting of adult brain tumors: initial experience. Am J Neuroradiol 38:492-499

3. Liao C, Wang K, Cao X, Li Y, Wu D, Ye H, Ding Q, He H, Zhong J (2018) Detection of lesions in mesial temporal lobe epilepsy by using MR fingerprinting. Radiology 288:804-812

4. Ma D, Jones SE, Deshmane A, Sakaie K, Pierre EY, Larvie M, McGivney D, Blümcke I, Krishnan B, Lowe M, Gulani V, Najm I, Griswold MA, Wang IZ (2018) Development of high-resolution 3D MR fingerprinting for detection and characterization of epileptic lesions. J Magn Reson Imaging 49:1333-1346

5. Sullivan DC, Obuchowski NA, Kessler LG, Raunig DL, Gatsonis C, Huang EP, Kondratovich M, Ane LM, Reeves AP, Barboriak DP, Guimaraes AR, Wahl RL, the Group F (2015) Metrology standards for quantitative imaging biomarkers. Radiology 277:813-825

6. of (ESR) E (2015) Magnetic resonance fingerprinting - a promising new approach to obtain standardized imaging biomarkers from MRI. Insights Imaging 6:163-165

7. Panda A, Mehta BB, Coppo S, Jiang Y, Ma D, Seiberlich N, Griswold MA, Gulani V (2017) Magnetic resonance fingerprintingan overview. Curr Opin Biomed Eng 3:56-66

8. Jiang Y, Ma D, Seiberlich N, Gulani V, Griswold MA (2014) MR fingerprinting using fast imaging with steady state precession (FISP) with spiral readout. Magnet Reson Med 74:1621-1631

9. Jiang Y, Ma D, Keenan KE, Stupic KF, Gulani V, Griswold MA (2017) Repeatability of magnetic resonance fingerprinting T1 and
T2 estimates assessed using the ISMRM/NIST MRI system phantom. Magn Reson Med 78:1452-1457

10. Körzdörfer G, Kirsch R, Liu K, Pfeuffer J, Hensel B, Jiang Y, Ma D, Gratz M, Bär P, Bogner W, Springer E, Cardoso P, Umutlu L, Trattnig S, Griswold M, Gulani V, Nittka M (2019) Reproducibility and repeatability of MR fingerprinting relaxometry in the human brain. Radiology 292:429-437

11. Tan H, Mayer C (2011) K-space trajectory estimation in spiral MRI system and related method thereof. US Patent No 7:888,935

12. Körzdörfer G, Pfeuffer J, Kluge T, Gebhardt M, Hensel B, Meyer CH, Nittka M (2019) Effect of spiral undersampling patterns on FISP MRF parameter maps. Magn Reson Imaging 62:174-180

13. Chung S, Kim D, Breton E, Axel L (2010) Rapid B1+ mapping using a preconditioning RF pulse with TurboFLASH readout. Magnet Reson Med 64:439-446

14. Koo TK, Li MY (2016) A guideline of selecting and reporting intraclass correlation coefficients for reliability research. J Chiropr Medicine 15:155-163

15. Schober P, Boer C, Schwarte LA (2018) Correlation coefficients. Anesth Analg 126:1763-1768

16. Chen Y, Jiang Y, Pahwa S, Ma D, Lu L, Twieg MD, Wright KL, Seiberlich N, Griswold MA, Gulani V (2016) MR fingerprinting for rapid quantitative abdominal imaging. Radiology 279:278-286

17. Kobayashi Y, Terada Y (2018) Diffusion-weighting caused by spoiler gradients in the fast imaging with steady-state precession sequence may lead to inaccurate T2 measurements in MR fingerprinting. Magn Reson Med Sci 18:96-104

18. Kessler LG, Barnhart HX, Buckler AJ, Choudhury K, Kondratovich MV, Toledano A, Guimaraes AR, Filice R, Zhang Z, Sullivan DC, Group TQ (2014) The emerging science of quantitative imaging biomarkers terminology and definitions for scientific studies and regulatory submissions. Stat Methods Med Res 24:9-26

19. Ye H, Cauley SF, Gagoski B, Bilgic B, Ma D, Jiang Y, Du YP, Griswold MA, Wald LL, Setsompop K (2016) Simultaneous multislice magnetic resonance fingerprinting (SMS-MRF) with directspiral slice-GRAPPA (ds-SG) reconstruction. Magnet Reson Med 77:1966-1974

20. Liao C, Bilgic B, Manhard M, Zhao B, Cao X, Zhong J, Wald LL, Setsompop K (2017) 3D MR fingerprinting with accelerated stack-of-spirals and hybrid sliding-window and GRAPPA reconstruction. Neuroimage 162:13-22

21. Cline CC, Chen X, Mailhe B, Wang Q, Pfeuffer J, Nittka M, Griswold MA, Speier P, Nadar MS (2017) AIR-MRF: accelerated iterative reconstruction for magnetic resonance fingerprinting. Magn Reson Imaging 41:29-40

22. da Cruz G, Bustin A, Jaubert O, Schneider T, Botnar RM, Prieto C (2019) Sparsity and locally low rank regularization for MR fingerprinting. Magnet Reson Med 81:3530-3543

23. Hoppe E, Körzdörfer G, Würfl T, Wetzl J, Lugauer F, Pfeuffer J, Maier A (2017) Deep learning for magnetic resonance fingerprinting: a new approach for predicting quantitative parameter values from time series. Stud Health Technol 243:202-206

Publisher's Note Springer Nature remains neutral with regard to jurisdictional claims in published maps and institutional affiliations. 